\title{
The Food and Health Project, a nutrition education intervention, increases fruit and vegetable awareness and intake among disadvantaged groups
}

\author{
S. Kleemann ${ }^{1}$, C. Cunningham ${ }^{1}$, C. Glennon Slattery ${ }^{1}$ and R. McEvoy ${ }^{2}$ \\ ${ }^{1}$ Community Nutrition and Dietetic Service, Health Service Executive Dublin Mid Leinster, Marlinstown Office Park, \\ Mullingar, Co. Westmeath, Republic of Ireland and ${ }^{2}$ Clinical Audit Department, William Street, Health Service Executive \\ Dublin Mid Leinster, Tullamore, Co. Offaly, Republic of Ireland
}

The dietary patterns of individuals in low-socio-economic groups are important as there is an inverse relationship between dietary quality and prevalence of chronic disease ${ }^{(1)}$. A partnership programme, 'The Food and Health Project', was set up in the Midland region of Ireland between the Health Service Executive, Dublin Mid Leinster and Westmeath Community Development Ltd in 1999. The project's purpose is to increase nutritional knowledge and improve eating practice and develop healthy and practical cooking skills among disadvantaged groups. A six-session course is delivered over a 6-week time-frame by peer instructors to groups of adult men and women by means of a peer-led nutrition education programme.

The aim of the present study was to determine participants' knowledge, pre- and post intervention, regarding the recommendation to consume five portions of fruit and vegetables daily. A structured questionnaire was used to collect data; the questionnaire was administered on the first day of the 6-week intervention programme under the supervision of the peer-led educator and again on the final day of the programme.

A total of sixteen open and closed questions were included in the pre-intervention questionnaire and twenty-one questions in the postintervention questionnaire. The questionnaire was approved by the National Adult Literacy Association of Ireland and also piloted amongst health professionals and previous course participants. Pre- and post intervention 101 questionnaires were completed and submitted for analysis. Data from the questionnaires were analysed using SPSS software v.14 (SPSS Inc., Chicago, IL, USA). Generally, results presented draw on basic descriptive statistics such as frequency and percentage tables for each on the questionnaire.

On average, participants consumed 3.9 portions of fruit and vegetables daily before the intervention. About one-quarter of the participants consumed on average less than one portion in total of fruit and vegetables daily. Awareness of the national nutritional recommendation for fruit and vegetables increased from $25.5 \%$ pre-intervention to $53 \%$ post intervention. This increased knowledge was reflected in the participants' behaviour, as fruit and vegetable consumption increased by $18.4 \%$ post intervention. The Table shows the change in daily fruit and vegetable consumption over the 6 week time-frame.

\begin{tabular}{lccc}
\hline & Portions of fruit & Portions of vegetable & Total no. of portions \\
\hline Pre-intervention & 1.90 & 2.03 & 3.94 \\
Post intervention & 2.35 & 2.55 & 4.90 \\
\hline
\end{tabular}

The data suggest that peer-led nutrition interventions such as The Food and Health Project can achieve positive changes in awareness and intake of fruit and vegetables among disadvantaged groups. The study is part of an ongoing process of nutrition education programmes delivery to disadvantaged groups in the Midland region of Ireland.

1. Kant AK, Schatzkin A, Graubard BI \& Schairer C (2000) JAMA 283, 2109-2115. 